\title{
Ultrasonic Needles for Bone Biopsy
}

\author{
Andrew Mathieson, Robert Wallace, Rebecca Cleary, Li Li, Hamish Simpson, and Margaret Lucas
}

\begin{abstract}
Bone biopsy is an invasive clinical procedure, where a bone sample is recovered for analysis during the diagnosis of a medical condition. When the architecture of the bone tissue is required to be preserved, a core-needle biopsy is taken. Although this procedure is performed while the patient is under local anaesthesia, the patient can still experience significant discomfort. Additionally, large haematoma can be induced in the soft tissue surrounding the biopsy site due to the large axial and rotational forces, which are applied through the needle to penetrate bone. It is well documented that power ultrasonic surgical devices offer the advantages of low cutting force, high accuracy, and preservation of soft tissues. This paper reports a study of the design, analysis, and test of two novel power ultrasonic needles for bone biopsy that operate using different configurations to penetrate bone. The first utilizes micrometric vibrations generated at the distil tip of a full-wavelength resonant ultrasonic device, while the second utilizes an ultrasonic-sonic approach, where vibrational energy generated by a resonant ultrasonic horn is transferred to a needle via the chaotic motion of a free-mass. It is shown that the dynamic behavior of the devices identified through experimental techniques closely match the behavior calculated through numerical and finiteelement analysis methods, demonstrating that they are effective design tools for these devices. Both devices were able to recover trabecular bone from the metaphysis of an ovine femur, and the biopsy samples were found to be comparable to a sample extracted using a conventional biopsy needle. Furthermore, the resonant needle device was also able to extract a cortical bone sample from the central diaphysis, which is the strongest part of the bone, and the biopsy was found to be superior to the sample recovered by a conventional bone biopsy needle.
\end{abstract}

Index Terms-Bone biopsy, dynamic model, finite element model, surgical device, ultrasonic needle.

\section{INTRODUCTION}

A BIOPSY involves the extraction of a sample of tissue from the body. Biopsies can be recovered from any part of the body, and are often extracted from the site of suspected disease to aid diagnosis. For example, if a musculoskeletal tumor is suspected, a biopsy is required to confirm this diagnosis after considered clinical evaluation and imaging analysis [1]. When a disease affects the whole body, such as leukaemia (cancer of the white blood cells), osteoporosis (bone weakening due a loss of bone), and renal osteodystrophy

Manuscript received December 21, 2015; accepted November 22, 2016. Date of publication December 7, 2016; date of current version February 1, 2017. This work was supported by the Engineering and Physical Sciences Research Council (EPSRC) EP/K020013/1. (Corresponding author: Margaret Lucas.)

A. Mathieson, R. Cleary, L. Li, and M. Lucas are with the School of Engineering, University of Glasgow, Glasgow G12 8QQ, U.K. (e-mail: margaret.lucas@glasgow.ac.uk).

R. Wallace and H. Simpson are with the Department of Orthopaedics and Trauma, University of Edinburgh, Edinburgh EH16 4SB, U.K.

Digital Object Identifier 10.1109/TUFFC.2016.2633286 (a weakening of bone tissue due to kidney disease), a biopsy site that eases clinical access and results in minimum discomfort to the patient is selected. Locations, where soft tissue is minimal and bone is thin, such as the iliac crest and the sternum, are typically accessed.

Bone biopsies can be performed by a fine-needle (biopsy and aspirate) or by a core-needle (trephine). Of these two types, the core-needle biopsy is the more invasive [2] but, unlike the biopsy and aspirate technique, it is capable of penetrating a thick layer of bone. Additionally, the biopsy and aspirate technique is not suitable for all diagnoses. In a bone lesion biopsy, where a biopsy is taken of a suspected musculoskeletal tumour, core-needle biopsies should be extracted as these preserve the architecture of the tissue, allowing immunohistochemistry to be used to aid diagnosis [3].

Core-needle biopsy is a routine procedure, typically over 1000 per annum are carried out in large regional centers, with approximately 100-200 per annum in district general hospitals [4]. Due to its invasiveness, it is often performed under local anaesthesia [5], regularly with additional pain relief [6]. Despite these measures, most patients still report high levels of pain during the biopsy, as well as residual pain, which can last for three to four days postprocedure [2]. In addition to the discomfort experienced by the patient during penetration of the bone, extracting a sample using a trephine needle can induce a large haematoma in the soft tissue surrounding the biopsy site. This is a result of the large forces and needle movements required during a biopsy, which can also limit the accuracy of the biopsy.

Power ultrasonic surgical devices have been shown since the 1950s to offer greater precision, protect soft tissue, reduce mechanical damage, and lower forces applied to the surgical site compared with conventional bone burs or saws [7]-[15]. Despite these advantages, it was not until the turn of the 21st century that power ultrasonic devices were routinely used to cut or penetrate mineralized tissue [15], where they are particularly advantageous for cutting delicate bone structures, where high precision is necessary. Consequently, ultrasonic devices have been increasingly adopted in oral and maxillofacial surgeries, neurosurgery, and orthopaedic procedures [14], [15].

The main challenges associated with core-needle bone biopsy, where bone microarchitecture must be preserved, for instance when immunohistochemistry is required, are achieving low needle force and high precision. To meet these challenges, this paper reports two novel power ultrasonic bone biopsy needles, which utilize completely different vibrational behaviors in order to penetrate bone and recover a biopsy sample. Their distinctive vibrational characteristics are illustrated 


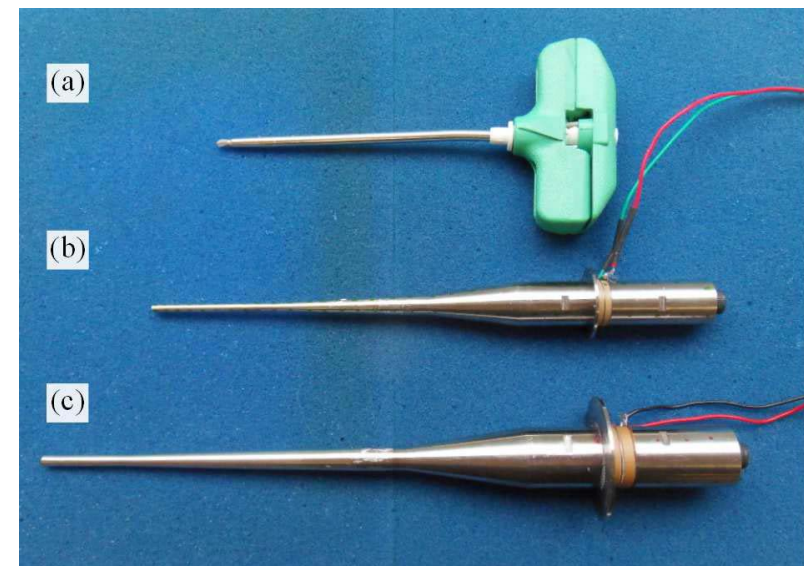

Fig. 1. (a) Trephine bone biopsy needle. (b) USR needle 1. (c) USR needle 2.

and, subsequently, their capability to recover biopsy samples from ovine bone is demonstrated and compared with existing techniques.

\section{Biopsy NeEDLES}

\section{A. Core-Needle Biopsy}

A core-needle (trephine) [Fig. 1(a)] is a conventional clinical instrument, which is presently used to penetrate hard tissue or to recover a tissue sample within which the architecture has to be preserved. To achieve this, the trephine biopsy needle possesses a cutting surface on its leading edge, which allows the clinician to penetrate through bone by a combined application of a large force in the direction of travel and an intermittent repetitive twisting motion. In practice, the twisting motion can have a relatively large conical orbit, creating a hole, which is larger than the diameter of the needle, and often causing damage to the soft tissue, which can increase the resulting haematoma from the procedure.

\section{B. Ultrasonic Needle Configurations}

Two different approaches are proposed for the design of alternative biopsy needles that rely on ultrasonic excitation. Figs. 1(b) and (c) and 2(a) present the architecture of the ultrasonic resonant (USR) needle. A half-wavelength Langevin transducer is connected via a threaded joint to a halfwavelength resonant needle, where $L_{\mathrm{Con}}$ and $L_{\mathrm{UF}}$ represent the sections of the needle insert with a conical and uniform profile, respectively. The USR needle is designed to operate in the second longitudinal mode of vibration. The micrometric vibrations generated at the distil tip of the needle combined with a very slow $120^{\circ}$ twisting action superimposed by the operator are sufficient for the needle to penetrate bone. Two USR needles, referred to as needle 1 and needle 2 in this paper, were designed based on different Langevin transducers and needles of different diameter.

The ultrasonic-sonic (USS) needle, shown in Fig. 2(b) and 8, is based on USS drills originally developed for drilling rock in low-gravity environments [16], [17]. This needle device consists of five main components; a Langevin transducer, a resonant stepped horn, a free-mass, a biopsy needle, and a spring. Ultrasonic vibrations generated at the distil tip of the stepped horn drive a free-mass, which vibrates chaotically

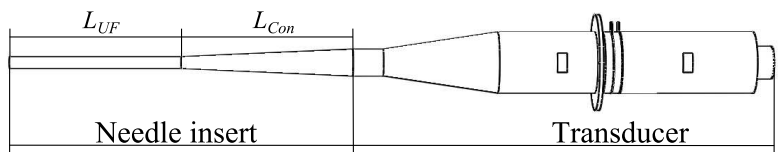

(a)

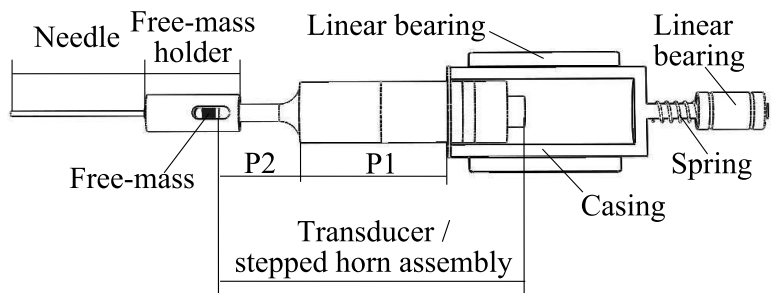

(b)

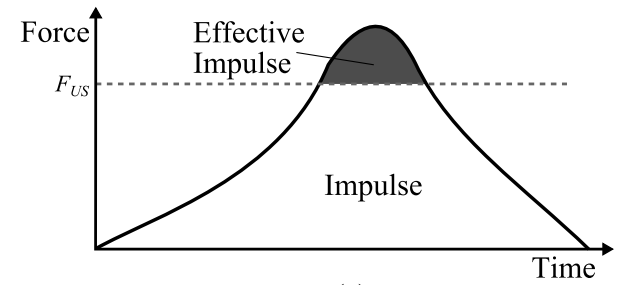

(c)

Fig. 2. Schematic of (a) USR needle and (b) USS needle. (c) Illustration of effective impulse.

at sonic frequencies, transferring energy to the needle. When the velocity of the distil tip of the horn is at its highest, this maximizes momentum transfer from the horn to the free-mass. The momentum of the free-mass is transferred to the needle on collision, which subsequently applies an impulse to the bone. It is known from the studies of rock drilling and coring that the rate of penetration in rock is directly proportional to the area under the applied force-time curve when the cutting tool (in this case needle) drives into the target material [17]. Bone is a highly viscoelastic material, whose strength increases with increased strain rate [18]. For the needle to penetrate bone, the force applied to the target site should be greater than required to reach the ultimate strength of bone (the stress threshold before bone failure occurs). An effective impulse can be identified above the force threshold, $F_{\mathrm{US}}$, as shown in Fig. 2(c), and is the impulse that allows progress to be made in penetrating the bone. A spring is incorporated in the device as shown in Fig. 2(b) to deliver a small force that pushes the needle against the target, while linear bearings allow the device to move freely during operation.

\section{Ultrasonic Biopsy NeEdle Design AND CHARACTERIZATION}

\section{A. Experimental Techniques}

The resonant frequencies and modes of vibration of the USR needles and of the transducer-horn assembly of the USS needle were modeled using the finite-element analysis (FEA) software, Abaqus (Dassault Systèmes). The FEA predictions were validated using experimental modal analysis (EMA), which has been described previously [19]. To ensure that the ultrasonic transducer-horn assemblies were driven at a constant vibration amplitude and at resonance, LabVIEW software (National Instruments) was used to coordinate a resonance 


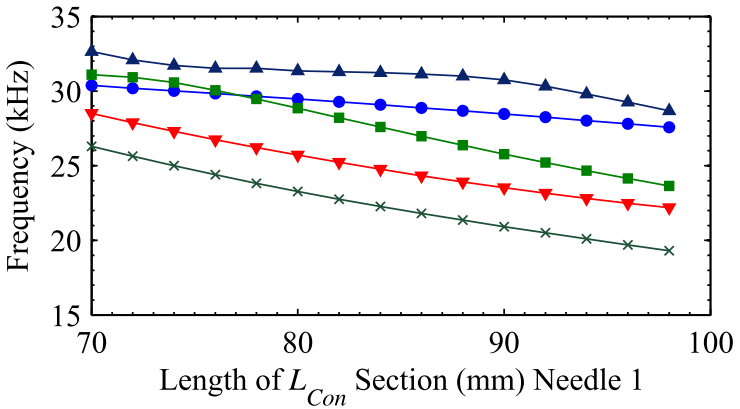

(a)

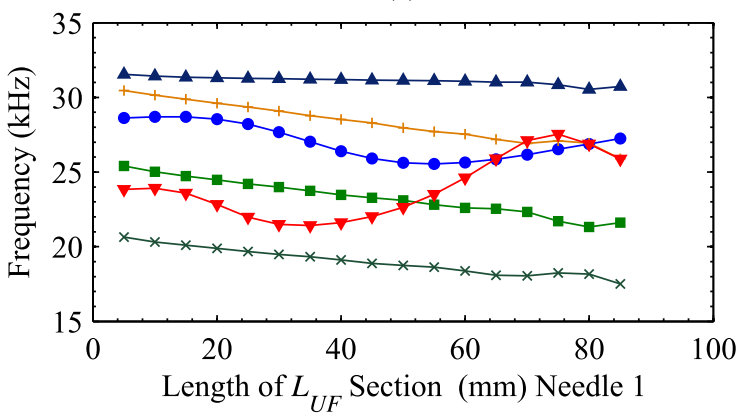

(b)

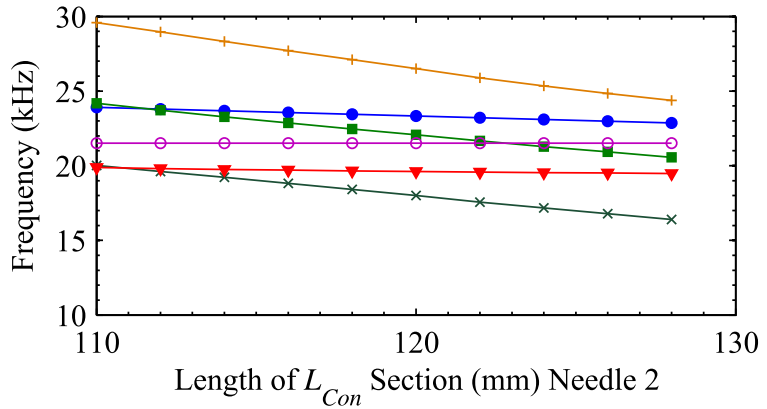

(c)

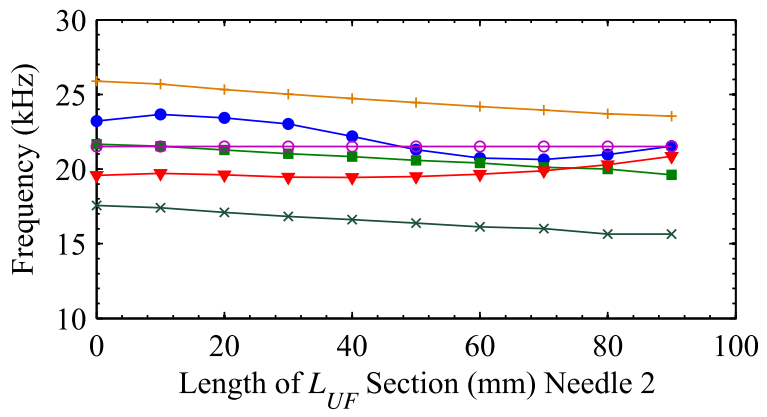

(d)

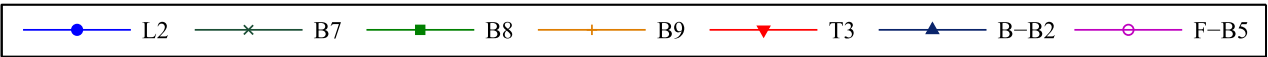

Fig. 3. Predicted modal frequencies of the transducer-needle assemblies as (a) $F_{\text {Con }}$ is adjusted in USR needle 1 , (b) $L_{\text {UF }}$ is adjusted in USR needle 1, (c) $F_{\text {Con }}$ is adjusted in USR needle 2, and (d) $L_{U F}$ is adjusted in USR needle 2, where L2: second longitudinal mode of vibration, T3: third torsional mode of vibration, B-B2: second bending of mode of the preload bolt, F-B5: fifth bending mode of the flange of the transducer, and B7, B8, and B9: seventh, eighth, and ninth bending mode of vibration.

tracking protocol [20]. The resonance tracking system is interfaced with a function generator (33210A, Aglient Technologies) using a data acquisition (DAQ) system (PXI-5922, NI) to generate the excitation signal, which was amplified by a power amplifier (2200L, E\&I). Current and voltage probes (P6022 and P139A, Tekronix) were used to feed the excitation signal back to the tracking system.

\section{B. Ultrasonic Resonant Needle}

1) USR Needle Configuration: The USR needles are fullwavelength resonant devices tuned to operate in the second longitudinal mode of vibration [Figs. 1 and 2(a)]. Both devices contain a $316 \mathrm{~L}$ stainless steel needle insert connected to a Langevin transducer via a threaded joint. The Langevin transducer in USR needle 1 contains two Sonox P8 (CeramTec UK) piezoceramic rings (outer diameter (OD): $16 \mathrm{~mm}$, inner diameter (ID): $7.2 \mathrm{~mm}$, and thickness: $2 \mathrm{~mm}$ ) sandwiched between Ti6Al4V endmasses. USR needle 2 also incorporates a Langevin transducer with Ti6Al4V endmasses and two Sonox P8 piezoceramic rings (OD: $20 \mathrm{~mm}$, ID: 8.2 $\mathrm{mm}$, and thickness: $4.06 \mathrm{~mm}$ ), while the internal diameters of the needle insert for USR needles 1 and 2 are 1.8 and $2.9 \mathrm{~mm}$, respectively.

2) Device Tuning and Modal Identification: It is known that slender multiple half-wavelength power ultrasonic devices can exhibit undesirable vibrational behaviors, such as modal coupling, which can detrimentally affect their performance and reliability [19]. To minimize the likelihood of such behaviors, the USR needles were tuned to maximize the modal frequency separation between the second longitudinal mode of vibration and neighboring modes of vibration.

During device tuning, the needle inserts of both USR needles initially possessed only a conical profile of length $F_{\text {Con }}$. This profile reduced from an OD of $7 \mathrm{~mm}$ to an OD of $3 \mathrm{~mm}$ for USR needle 1 and an OD of $8 \mathrm{~mm}$ to an OD of $4 \mathrm{~mm}$ for USR needle 2. Fig. 3(a) and (c) presents the FEA-predicted resonant frequencies of these two devices as the length of the needle insert increases from 70 to $98 \mathrm{~mm}$ for USR needle 1 and 110 to $128 \mathrm{~mm}$ for USR needle 2. For USR needle 1, a needle length of $90 \mathrm{~mm}$ offers the largest modal frequency separations ( $8 \%$ and $9 \%$ ) between the second longitudinal mode of vibration and the neighboring modes of vibration, namely the second bending mode of the prestress bolt and the eighth bending mode of the device. The comparable needle length for USR needle 2 was $122 \mathrm{~mm}$, providing the largest modal frequency separations (9\% and $9 \%$ ) between the second longitudinal mode of vibration and the neighbouring eighth and ninth bending modes.

The section of the needle inserts that penetrate soft tissues and bone, $L_{\mathrm{UF}}$, possesses an uniform outer diameter of 3 and $4 \mathrm{~mm}$, for USR needle 1 and USR needle 2, respectively. These are consistent with gauge 11 and 8 standard biopsy needles used in clinical practice. The length of USR needle 1 was fixed at $90 \mathrm{~mm}$, while USR needle 2 was fixed at $122 \mathrm{~mm}$, in line with the results of the FE analysis. To ensure that the uniform length of the needle, $L_{\mathrm{UF}}$, did not induce undesirable vibrational behavior, $L_{\mathrm{UF}}$ was adjusted, until the frequency separations between the second 


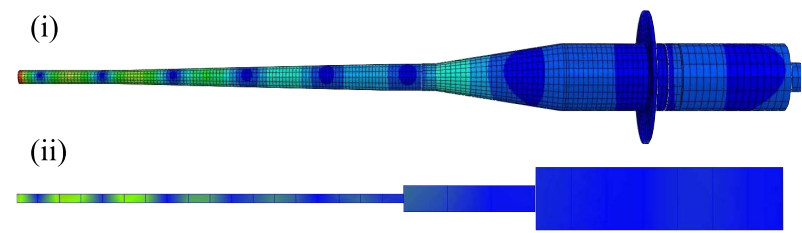

(a)

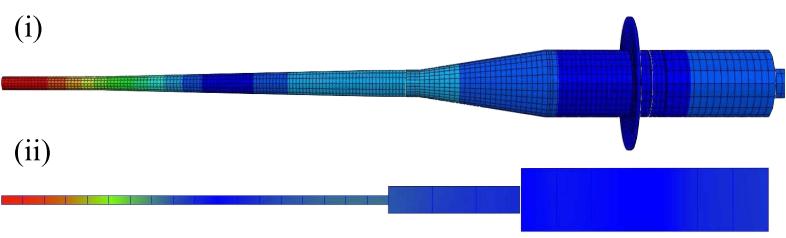

(b)

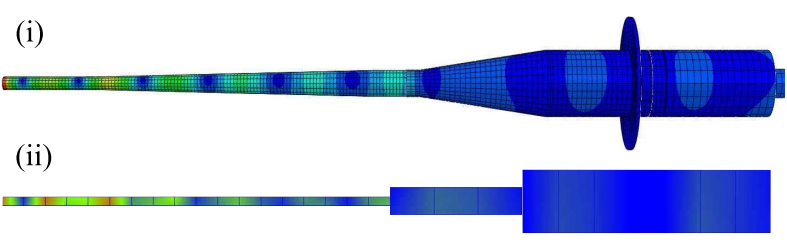

(c)

Fig. 4. Mode shapes identified from USR needle 2. (i) FEA. (ii) EMA (geometry representing the measurement grid). (a) Eighth bending, (b) second longitudinal, and (c) ninth bending modes of vibration. Blue through to red represents contours of low to high displacement amplitude.

TABLE I

RESonANT FREQUENCIES OF OPERATIONAL AND NEIGHBouring Modes of Vibration Predicted Through FEA AND MEASUREd Via EMA

\begin{tabular}{lccc} 
& $\begin{array}{c}f_{F E A} \\
(\mathrm{~Hz})\end{array}$ & $\begin{array}{c}f_{E M A} \\
(\mathrm{~Hz})\end{array}$ & $\begin{array}{c}\Delta f_{F E A-E M A} \\
(\%)\end{array}$ \\
\hline USR needle 1 & & & \\
$8^{\text {th }}$ Bending & 23298 & 23312 & 0.1 \\
$2^{\text {nd }}$ Longitudinal & 25907 & 25687 & 0.8 \\
$9^{\text {th }}$ Bending & 28290 & 27809 & 1.7 \\
\hline USR needle 2 & & & \\
$8^{\text {th }}$ Bending & 21033 & 20865 & 0.8 \\
$2^{\text {nd }}$ Longitudinal & 23024 & 23515 & 2.1 \\
$9^{\text {th }}$ Bending & 25022 & 24573 & 1.8
\end{tabular}

longitudinal mode and its neighboring modes of vibration were maximized.

Fig. 3(b) and (d) shows the predicted resonant frequencies of the USR needles with varying $L_{\mathrm{UF}}$. The largest modal frequency spacings (10\% and $9 \%)$ between the second longitudinal mode and the eighth and ninth bending modes were predicted for $L_{\mathrm{UF}}=45 \mathrm{~mm}$. For USR needle 2, the frequency spacings were largest $\left(9 \%\right.$ and $16 \%$ ) for $L_{U F}=30 \mathrm{~mm}$.

EMA was used to identify the resonant frequencies of the USR needles and to extract the corresponding mode shapes. Table I and Fig. 4 illustrate that the frequencies and mode shapes extracted from EMA closely match the predicted resonant frequencies and mode shapes. The curve-fitted frequency response functions (FRFs) of the USR needles are shown in Fig. 5. High modal density is observed in the FRFs, and this can be accredited to the hollow and slender profile of the needle inserts. However, there is sufficient frequency spacing to ensure that modal coupling does not occur. This is confirmed by the absence of a parasitic vibrational response in the extracted mode shape of the second longitudinal mode (Fig. 4),

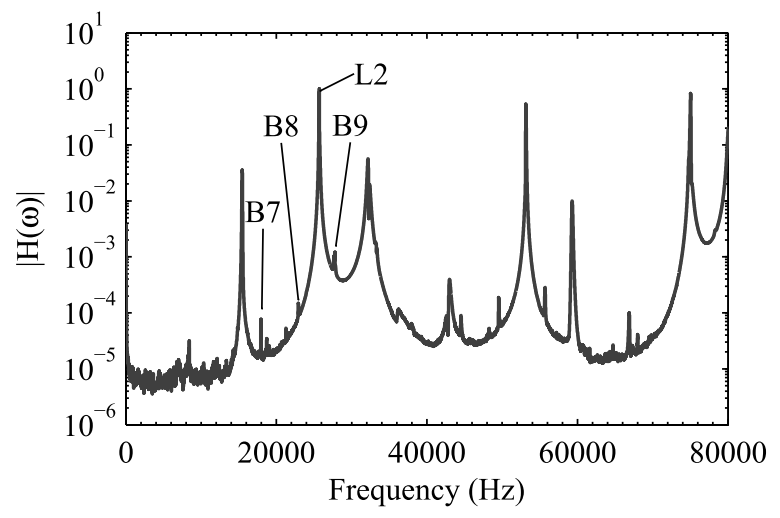

(a)

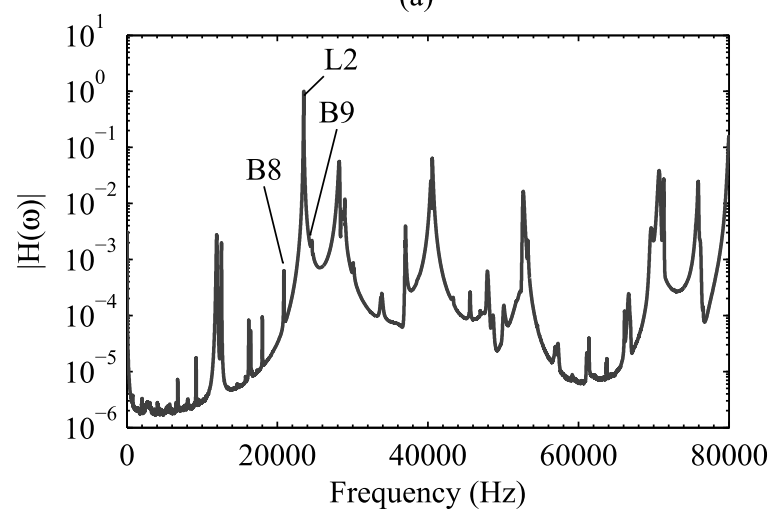

(b)

Fig. 5. Curve-fitted FRFs extracted from EMA. (a) USR 1. (b) USR 2.

which is measured to vibrate with only a longitudinal response.

\section{Ultrasonic-Sonic Needle}

1) Stepped Horn Configuration: USS drills developed for rock drilling applications are full-wavelength devices, which are tuned to operate at approximately $20 \mathrm{kHz}$ [16], [17]. To reduce the size for application in clinical procedures, the USS system has been tuned to operate longitudinally at $50 \mathrm{kHz}$. The half-wavelength Langevin transducer, which has a diameter of $20 \mathrm{~mm}$ and a length of $42.1 \mathrm{~mm}$ and contains two Ceramtec P8 piezoceramic rings (OD: $20 \mathrm{~mm}$, ID: $8 \mathrm{~mm}$, and thickness: $4 \mathrm{~mm}$ ), is connected to a halfwavelength stepped horn, which amplifies the micrometric vibrations generated by the transducer.

Needle penetration rate is dependent on momentum transfer from the resonant ultrasonic device to the needle via the chaotic motion of the free-mass. This is influenced by the vibrational amplitude of the distil tip of the ultrasonic horn and its effective mass, which is defined as the part of the horn tip that influences the dynamic behavior of the free-mass during an impact event [16]. The amplitude gain of the stepped horn is determined by the ratio of its base diameter and distil tip diameter. Although a large diameter ratio leads to a highgain horn, a small distil tip diameter can detrimentally affect the effective mass of the horn, and can also induce elevated stresses in the horn.

To determine the diameter ratio that maximizes momentum transfer to the free-mass, stepped horns with a range of distil 


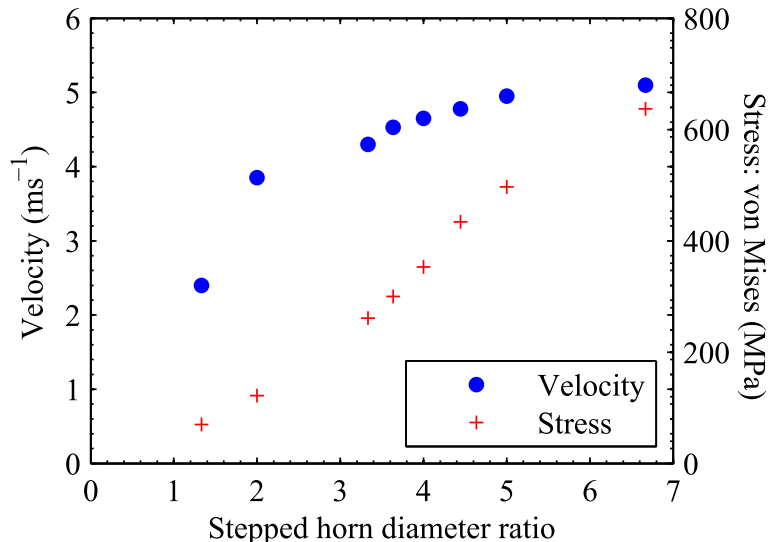

Fig. 6. Free-mass velocity and maximum stress in the horn, predicted via FEA.

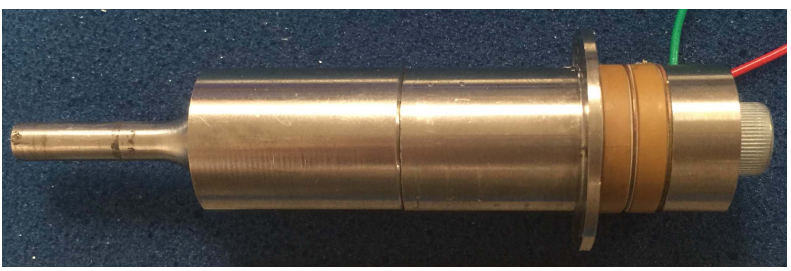

(a)

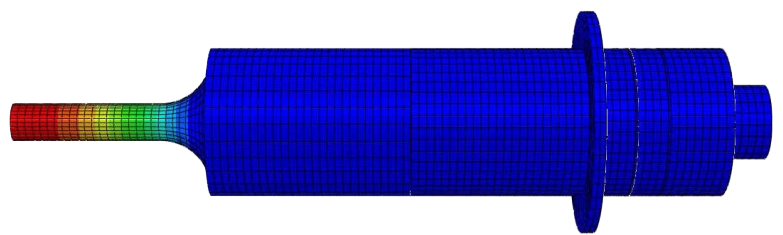

(b)

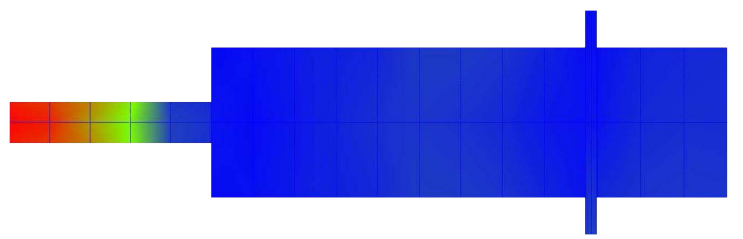

(c)

Fig. 7. (a) USS transducer-horn assembly. (b) FEA: mode shape of operational mode of vibration. (c) EMA: mode shape of operational mode of vibration (geometry representing the measurement grid). Blue through to red represents contours of low to high displacement amplitude.

tip diameters were modeled impacting the same free-mass using FEA. From Fig. 6, it can be seen that the highest free-mass velocity is predicted for the horn with the largest diameter ratio. This indicates that the vibrational amplitude of the stepped horn dominates momentum transfer. However, the maximum stress predicted in this stepped horn $(637 \mathrm{MPa})$ is significantly greater than the estimated fatigue limit $(410 \mathrm{MPa})$ of the alloy (Ti6V4Al) [21]. A stepped horn with a diameter ratio of 3.6 (5.5 $\mathrm{mm}$ distil tip diameter) was, therefore, selected and manufactured for the USS needle.

The resonant frequency of the operational mode of vibration (second longitudinal mode) of the transducer-stepped horn assembly was predicted using FEA at $50.56 \mathrm{kHz}$, and identified via EMA at $48.95 \mathrm{kHz}$, representing a $3.2 \%$ difference. Fig. 7 presents the corresponding mode shapes at the oper-

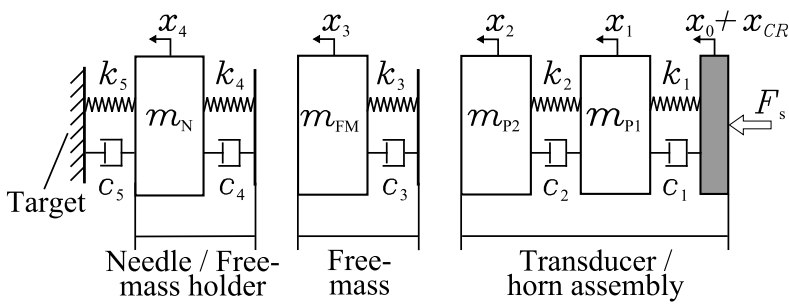

(a)

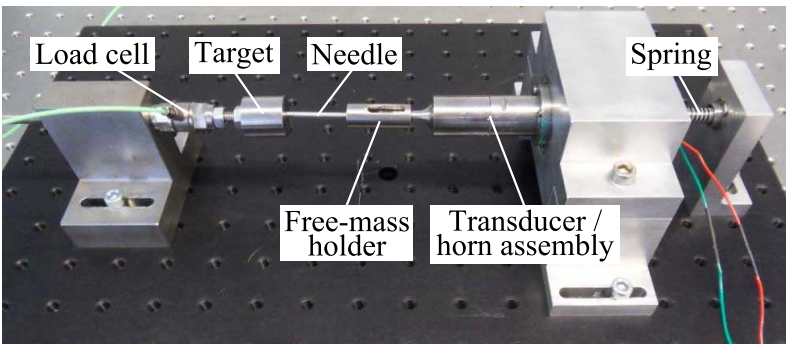

(b)

Fig. 8. (a) MSD model of the USS needle architecture. (b) Force measurement experimental setup.

ational resonant frequency, extracted from FEA and EMA showing a close match.

2) MSD Model and Experimental Validation: A massspring-damper (MSD) model of the USS needle configuration was developed to predict the dynamic behavior of the device. Predicting the force applied to the target site (or biopsy site) allowed the effective impulse to be estimated, which is an indicator of device performance. Altering free-mass and needle parameters allowed optimal configurations to be identified and the effective impulse maximized. Fig. 8(a) presents the MSD model, where $m, k$, and $c$ represent the effective mass, stiffness, and damping of the individual dynamic components. The force exerted by the spring is defined as $F_{S}$, while $x_{0}$ and $x_{\mathrm{CR}}$ represent the vibrational displacement of the piezoceramic stack and rear of the transducer, respectively. It can be approximated that the piezoceramic stack is located in the nodal plane of the longitudinal mode, and hence, it is at a location of zero displacement [22]. This allows the rear of the transducer, which encompasses the backmass, casing, and rearmost piezoceramic ring, to be included in the MSD expression, which describes the motion at the piezoceramic stack (1). Equation 2 expresses the motion of the transducer front mass and the part of the stepped horn with a diameter of $20 \mathrm{~mm}$, referred to as P1 in Fig. 2. Equations 3-5 represent the other part of the horn (P2) with a $5.5 \mathrm{~mm}$ diameter, the free-mass (FM), and needle $(N)$

$$
\begin{aligned}
m_{C R} \ddot{x}_{C R}= & F_{s}-F_{k 1}-F_{c 1} \\
= & F_{s}-\left(x_{C R}+x_{0}-x_{1}\right) k_{1} \\
& -\left(\dot{x}_{C R}+\dot{x}_{0}-\dot{x}_{1}\right) c_{1} \\
m_{P 1} \ddot{x}_{1}= & F_{k 1}+F_{c 1}-F_{k 2}-F_{c 2} \\
= & \left(x_{C R}+x_{0}-x_{1}\right) k_{1}+\left(\dot{x}_{C R}+\dot{x}_{0}-\dot{x}_{1}\right) c_{1} \\
& -\left(x_{1}-x_{2}\right) k_{2}-\left(\dot{x}_{1}-\dot{x}_{2}\right) c_{2} \\
m_{P 2} \ddot{x}_{2}= & F_{k 2}+F_{c 2}-F_{k 3}-F_{c 3} \\
= & \left(x_{1}-x_{2}\right) k_{2}+\left(\dot{x}_{1}-\dot{x}_{2}\right) c_{2} \\
& -\left(x_{2}-x_{3}\right) k_{3}-\left(\dot{x}_{2}-\dot{x}_{3}\right) c_{3}
\end{aligned}
$$




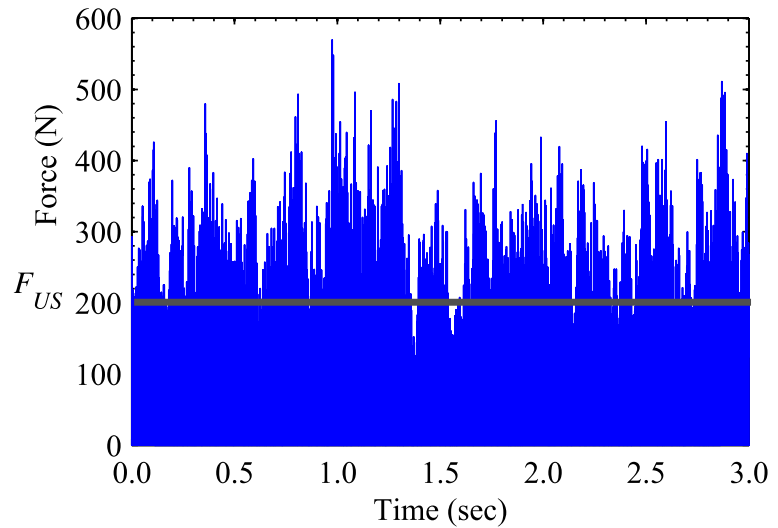

(a)

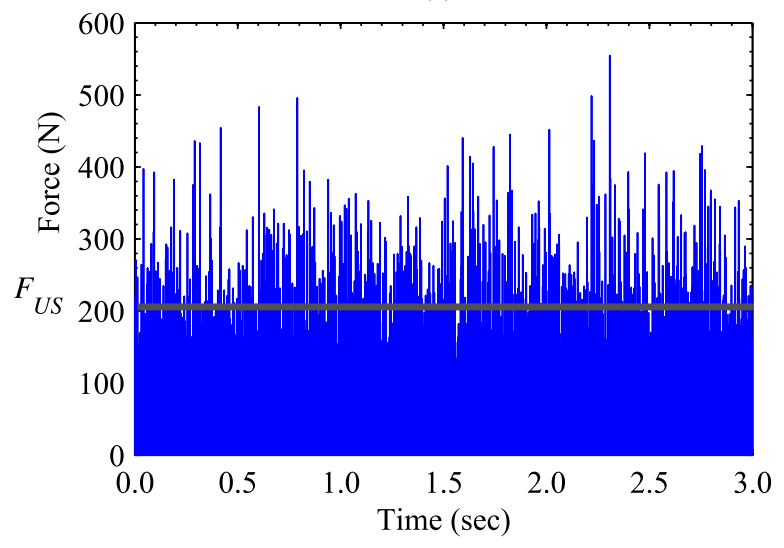

(b)

Fig. 9. Force-time response of USS needle. The solid horizontal line represents $F_{\text {US }}$ of cortical bone [18]. (a) MSD model prediction, and (b) measured by the load cell.

$$
\begin{aligned}
m_{F M} \ddot{x}_{3}= & F_{k 3}+F_{c 3}-F_{k 4}-F_{c 4} \\
= & \left(x_{2}-x_{3}\right) k_{3}+\left(\dot{x}_{2}-\dot{x}_{3}\right) c_{3} \\
& -\left(x_{3}-x_{4}\right) k_{4}-\left(\dot{x}_{3}-\dot{x}_{4}\right) c_{4} \\
m_{N} \ddot{x}_{4}= & F_{k 4}+F_{c 4}-F_{k 5}-F_{c 5} \\
= & \left(x_{3}-x_{4}\right) k_{4}+\left(\dot{x}_{3}-\dot{x}_{4}\right) c_{4} \\
& -x_{4} k_{5}-\dot{x}_{4} c_{5} .
\end{aligned}
$$

Fig. 9 presents the force response of the needle tip impacting the target predicted by the MSD model and measured using the experimental setup shown in Fig. 8(b). The force was measured by a load cell (Kistler 9311B) and corresponding force data were acquired using a DAQ interface (Picoscope 4424, Pico Technology). The free-mass used in the MSD model and experiment had a mass of $0.57 \mathrm{~g}$, diameter of $5.5 \mathrm{~mm}$, and length of $5.43 \mathrm{~mm}$. Both of the time domain traces demonstrate a chaotic force response, and both are for vibrational amplitude of $8 \mu \mathrm{m}$ at the distal tip of the ultrasonic horn. Discrepancies between the modeled and measured motion of the free-mass are visible in Fig. 9. Nevertheless, the magnitudes of the simulated and measured forces lie within the same force range and exceed $F_{\text {US }}$. The effective impulse was calculated using an estimation for the strength of ovine bone of $200 \mathrm{MPa}$ [18] and area of the needle tip was $1 \mathrm{~mm}^{2}$. Calculations were performed for three time durations of $1 \mathrm{~s}$ (first, second, and third) and
TABLE II

EFFECTIVE IMPUlSE CALCULATEd From MSD AND EXPERIMENTAL DATA

\begin{tabular}{lcc}
1 s duration & \multicolumn{2}{c}{ Effective Impulse (Ns/s) } \\
& MSD prediction & Measured \\
\hline $1^{\text {st }}$ & 0.47 & 0.31 \\
$2^{\text {nd }}$ & 0.53 & 0.32 \\
$3^{\text {rd }}$ & 0.47 & 0.35 \\
\hline Average & 0.49 & 0.33
\end{tabular}

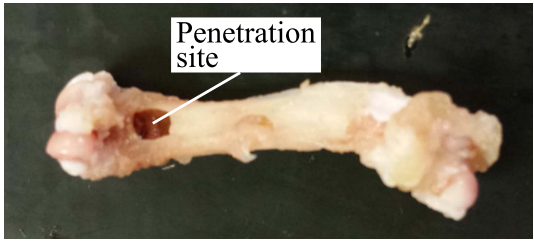

(a)

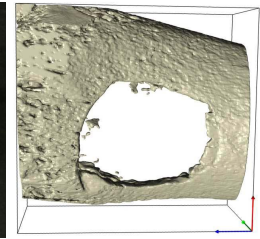

(b)
Fig. 10. Femur bone of Wister rat. (a) Penetration site. (b) $\mu \mathrm{CT}$ reconstruction of penetration site.

the results are presented in Table II. The chaotic nature of the motion of the free-mass is evident in the inconsistencies in the effective impulses in different 1-s periods. Although the MSD model currently overpredicts the effective impulse, the MSD has proven to be a reasonable predictive tool for the design of the USS needle device.

\section{BIOPSY TRIALS}

\section{A. $\mu$ CT Method}

The structural quality of the biopsy site and the samples recovered by the USR needles, USS needle, and a conventional trephine biopsy needle were assessed by microcomputed tomography $(\mu \mathrm{CT})$. This technique allows the internal structure of tissue to be assessed nondestructively. All cores were scanned through 360 degrees using a Skyscan 1172 (Bruker, Kontich, Belgium) with a voxel resolution of $2.93 \mu \mathrm{m}$ using the following settings; source voltage: $71 \mathrm{kV}$, source current: $139 \mu \mathrm{A}$, and exposure duration: $1178 \mathrm{~ms}$. To ensure highquality images, four frame averaging and a random movement value of 5 were used for all scans. After scanning, the images were reconstructed and analyzed using proprietary software (Nrec version 1.6.9.4, CTAn 1.13.5.1, and CTVox 2.6, all Bruker, Belgium).

\section{B. Biopsy Analysis: Wistar Rat Femur}

USR needle 1 successfully penetrated the femur of a threemonth old Wistar rat [Fig. 10(a)]. During this procedure, the femur was fixed into a bench-top vice. The USR needle was driven at a vibrational amplitude of $58 \mu \mathrm{m}$, while the operator applied a very low force, which was just sufficient to provide a good contact with the bone and to guide it through the biopsy site. Guidance and penetration were assisted by the operator superimposing a slow $120^{\circ}$ rotation while the needle advanced into the femur. To prevent tissue heating, a flow of phosphatebuffered saline (PBS) was delivered to the resonant needle and biopsy site at a rate of $20 \mathrm{ml} / \mathrm{min}$. It can be seen that USR needle 1 fully penetrated the cortical bone; however, no biopsy sample was recovered in this test. 


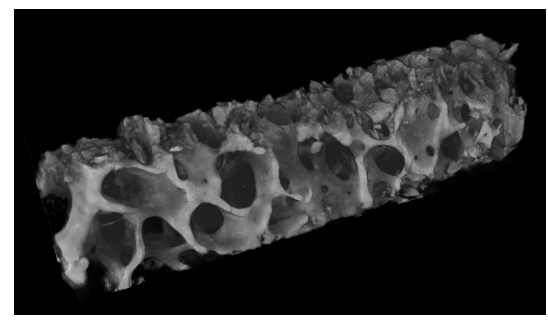

(a)

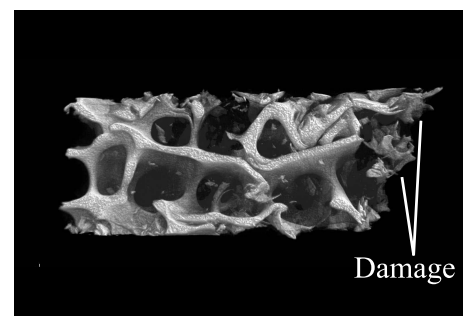

(b)

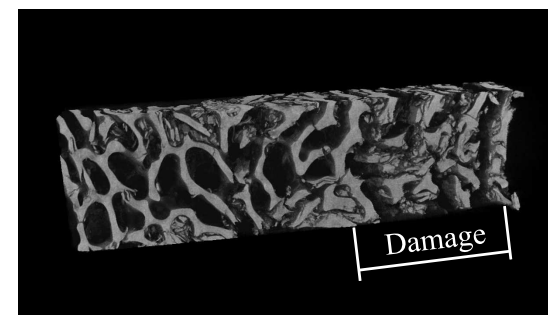

(c)

Fig. 11. Three-dimensional $\mu \mathrm{CT}$ reconstruction of metaphyseal tissue recovered by (a) trephine needle, (b) USS needle, and (c) USR needle 2.

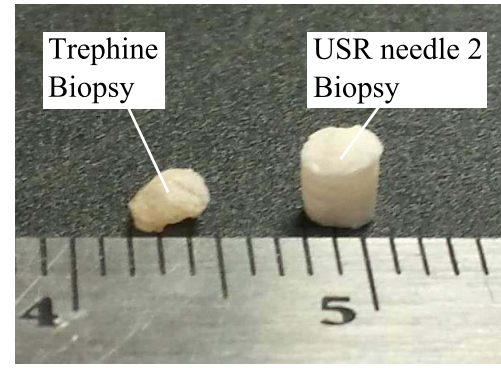

(a)

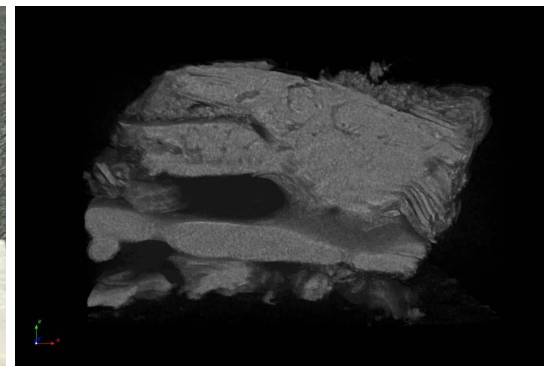

(b)

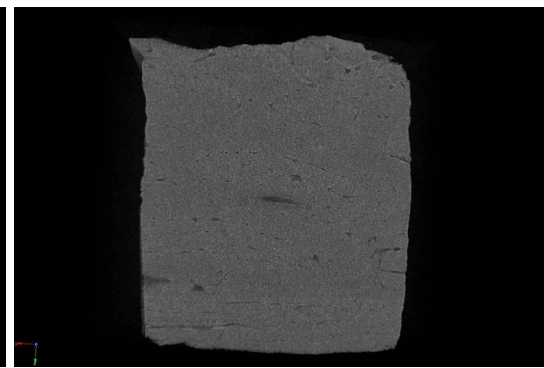

(c)

Fig. 12. Cortical bone tissue biopsy. (a) Photograph of biopsy samples; (b) $\mu \mathrm{CT}$ reconstruction of biopsy recovered by a trephine needle; and (c) $\mu \mathrm{CT}$ reconstruction of biopsy recovered by USR needle 2 .

The $\mu \mathrm{CT}$ reconstruction of the biopsy site [Fig. 10(b)] shows a small amount of microdamage around the penetration site. The irregular shape of the hole can be accredited to chipping, but no large cracks are visible. This is an encouraging result, as it is very difficult for a conventional trephine biopsy needle of similar diameter to penetrate a bone structure with a thin cortical diaphyses without inducing fracture.

\section{Biopsy Analysis: Ovine Metaphyseal Bone}

The USR needle 2 and the USS needle were both used to penetrate the ovine metaphysis, recovering biopsy samples (Fig. 11). The metaphysis refers to part of the bone, which lies between the diaphysis (shaft of the bone) and the epiphysis (the wider end of long bones). The test setup and needle penetration were consistent with the method used for USR needle 1, with the USR 2 needle driven at a vibrational amplitude of $80 \mu \mathrm{m}$. For the USS needle, the ovine femur was fastened to a custom clamp, which allowed the position of the femur to be adjusted. PBS was not required in this case. A trephine needle was also used to recover a biopsy sample using the conventional clinical penetration method, the femur being fixed in a bench-top vice.

Fig. 11 shows $\mu \mathrm{CT}$ reconstructed images of the metaphysis biopsies. Trabecular bone, also referred to as cancellous bone, typically found at the end of long bones, or in large bone structures, such as the pelvis and vertebrae, was extracted. From the $\mu \mathrm{CT}$ reconstruction, it can be seen that the trephine needle induced the least damage to the trabeculae. The damage at the end of the sample extracted by the USS needle [Fig. 11(b)] indicates that the sample broke away from the biopsy site during the procedure. Damage to the trabeculae can also be observed in one half of the sample extracted by USR needle 2 [Fig. 11(c)]. Nevertheless, the USS needle and
USR needle 2 both extracted intact and viable biopsy samples, where the majority of microarchitecture remained intact.

\section{Biopsy Analysis: Ovine Cortical Bone}

To further assess the capability of USR needle 2, it was used to recover a cortical bone sample from the central diaphysis of an ovine femur, the location where the bone is strongest. Cortical bone is much stronger, harder, and denser than trabecular [23], and hence is a more challenging tissue to recover a biopsy sample from. The experimental protocol used to extract a sample of ovine cortical bone using USR needle 2 was consistent with the protocol used to penetrate ovine metaphyseal. A trephine needle was also used to retrieve a biopsy. Fig. 12 shows biopsy samples of cortical bone extracted from the ovine femur using USR needle 2 and a trephine needle. It can be seen from Fig. 12(a) and (c) that the sample extracted by USR needle 2 is intact, has a uniform cylindrical profile, and the absence of chipping on the surface of the sample indicates that the needle penetrated with precision and accuracy. The profile of the sample also implies that the biopsy site was not subjected to significant stresses during the biopsy procedure. In this case, the profile of the biopsy sample extracted using the trephine needle has undergone significant deformation [Fig. 12(a) and (b)]. The shortened and twisted appearance of this sample indicates that it has been subjected to high axial and shear stress during its extraction. This stems from the high force $(>500 \mathrm{~N})$ and twisting action that the surgeon applies to the needle, and hence the femur, in order to recover a biopsy sample. Although the sample has been deformed, it is intact and could likely still be viable for diagnosis. However, it is clear that an ultrasonic bone biopsy needle is capable of extracting a significantly higher quality biopsy from even the most challenging bone sites. 


\section{CONCLUSION}

Two differently configured novel power ultrasonic bone biopsy needles have been presented. The dynamic behavior of the devices identified through experimental techniques closely matched the behavior predicted through numerical and FEA methods, demonstrating effective predictive capability for the design of ultrasonic needle devices.

It has been demonstrated that an ultrasonic needle device using either configuration (resonant needle or USS needle) is capable of recovering trabecular bone biopsies from the metaphysis of an ovine femur. The bone architectures of the samples were found to be largely intact, indicating the viability of the samples and their suitability for use in subsequent diagnosis. Although the trabecular bone biopsy sample recovered by the trephine was identified to be superior to the sample extracted by both ultrasonic device configurations, the quality of the samples are comparable. The resonant needle device was also used to extract a cortical bone sample from the central diaphysis of an ovine femur. Conversely, the cortical bone sample extracted by the trephine was significantly deformed, while the sample recovered using the ultrasonic device was a very uniform and undamaged cylindrical core. Importantly, the force required to ultrasonically extract a biopsy sample of trabecular or cortical bone is very small, only requiring to be sufficient to maintain good contact with the bone. The trephine needle, on the other hand, can require more than $500 \mathrm{~N}$ of penetration force combined with a damaging twisting action in order to penetrate cortical bone. It can be concluded that ultrasonic bone biopsy needle devices offer significant potential for replacing trephine needles for challenging bone biopsies.

\section{ACKNOWLEDGMENT}

The authors would like to acknowledge project partner Ethicon Inc.

\section{REFERENCES}

[1] J. Bickels, J. Jelinek, B. Shmookler, R. Neff, and M. Malawer, "Biopsy of musculoskeletal tumors: Current concepts," Clin. Orthopaedics Rel. Res., vol. 368, pp. 212-219, Nov. 1999.

[2] T. Valebjørg et al., "Pain and bleeding associated with trephine biopsy," Eur. J. Haematol., vol. 93, no. 4, pp. 267-272, Oct. 2014.

[3] C. Errani, F. Traina, F. Perna, C. Calamelli, and C. Faldini, "Current concepts in the biopsy of musculoskeletal tumors," Sci. World J., vol. 2013, 2013, Art. no. 538152.

[4] B. S. Wilkins and D. M. Clark, "Making the most of bone marrow trephine biopsy," Histopathology, vol. 55, no. 6, pp. 631-640, Dec. 2009.

[5] N. Hjortholm, E. Jaddini, K. Hałaburda, and E. Snarski, "Strategies of pain reduction during the bone marrow biopsy," Ann. Hematol., vol. 92, pp. 145-149, 2013.

[6] M. Zahid, "Methods of reducing pain during bone marrow biopsy: A narrative review," Ann. Palliative Med., vol. 4, no. 4, pp. 184-193, Oct. 2015.

[7] H. H. Postle, "Ultrasonic cavity preparation," J. Prosthetic Dentistry, vol. 8, no. 1, pp. 153-160, Jan. 1958.

[8] L. Balamuth, "Ultrasonics and dentistry," Sound, vol. 2, no. 2, pp. 15-19, 1963.

[9] H. Mazarow, "Bone repair after experimentally produced defects," $J$. Oral Surgery, Anesthesia, Hospital Dental Service, vol. 18, pp. 107$115,1960$.

[10] T. McFall, G. Yamane, and G. Burnett, "Comparison of the cutting effect on bone of an ultrasonic cutting device and rotary burs," J. Oral Surgery, Anesthesia, Hospital Dental Service, vol. 19, pp. 200-209, May 1961.
[11] J. Horton, T. Tarpley, and L. Wood, "The healing of surgical defects in alveolar bone produced with ultrasonic instrumentation, chisel, and rotary bur," Oral Surgery Oral Med. Oral Pathol., vol. 39, no. 4, pp. 536-546, Apr. 1975.

[12] H. Aro, H. Kallioniemi, A. Aho, and P. Kellokumpu-Lehtinen, "Ultrasonic device in bone cutting: A histological and scanning electron microscopical study," Acta Orthopaedica Scandinavica, vol. 52, no. 1, pp. $5-10,1982$.

[13] B. S. Khambay and A. D. Walmsley, "Investigations into the use of an ultrasonic chisel to cut bone. Part 2: Cutting ability," J. Dentistry, vol. 28 , no. 1, pp. 39-44, Jan. 2000.

[14] J.-L. Beziat, J.-C. Bera, B. Lavandier, and A. Gleizal, "Ultrasonic osteotomy as a new technique in craniomaxillofacial surgery," Int. J. Oral Maxillofacial Surgery, vol. 36, no. 6, pp. 493-500, Jun. 2007.

[15] M. Labanca, F. Azzola, R. Vinci, and L. F. Rodella, "Piezoelectric surgery: Twenty years of use," Brit. J. Oral Maxillofacial Surgery, vol. 46, no. 4, pp. 265-269, Jun. 2008.

[16] X. Bao et al., "Modeling and computer simulation of ultrasonic/sonic driller/corer (USDC)," IEEE Trans. Ultrason., Ferroelect., Freq. Control, vol. 50, no. 9, pp. 1147-1160, Sep. 2003.

[17] P. Harkness, M. Lucas, and A. Cardoni, "Maximization of the effective impulse delivered by a high-frequency/low-frequency planetary drill tool," IEEE Trans. Ultrason., Ferroelect., Freq. Control, vol. 58, no. 11, pp. 2387-2396, Nov. 2011.

[18] R. Wallace, P. Pankaj, and A. H. R. W. Simpson, "The effect of strain rate on the failure stress and toughness of bone of different mineral densities," J. Biomech., vol. 46, no. 13, pp. 2283-2287, Sep. 2013.

[19] A. Mathieson, A. Cardoni, N. Cerisola, and M. Lucas, "Understanding nonlinear vibration behaviours in high-power ultrasonic surgical devices," Proc. Roy. Soc. A, vol. 471, no. 2176, p. 20140906 , 2015.

[20] Y. Kuang, Y. Jin, S. Cochran, and Z. Huang, "Resonance tracking and vibration stablilization for high power ultrasonic transducers," Ultrasonics, vol. 54, no. 1, pp. 187-194, Jan. 2014.

[21] R. J. Morrissey and T. Nicholas, "Fatigue strength of Ti-6Al-4V at very long lives," Int. J. Fatigue, vol. 27, nos. 10-12, pp. 1608-1612, Oct./Dec. 2005

[22] S. Voronina and V. Babitsky, "Autoresonant control strategies of loaded ultrasonic transducer for machining applications," J. Sound Vibration, vol. 313 , nos. 3-5, pp. 395-417, Jun. 2008.

[23] D. R. Carter and W. C. Hayes, "The compressive behavior of bone as a two-phase porous structure," J. Bone Joint Surgery, vol. 59, no. 7, pp. 954-962, 1977.

Andrew Mathieson, photograph and biography not available at the time of publication.

Robert Wallace, photograph and biography not available at the time of publication.

Rebecca Cleary, photograph and biography not available at the time of publication.

$\mathbf{L i} \mathbf{L i}$, photograph and biography not available at the time of publication.

Hamish Simpson, photograph and biography not available at the time of publication.

Margaret Lucas, photograph and biography not available at the time of publication. 